\title{
LETTERS
}

\section{Hot tub lung: an occupational hazard}

\section{To the Editor:}

Hot tub baths have become increasingly common in residences as well as hotels and spa facilities. Recently, such baths have been associated with an emerging disorder known as hot tub lung (HTL). HTL is a diffuse granulomatous lung disease caused by inhalation of water aerosol containing non-tuberculous mycobacteria (NTM), in most cases belonging to the Mycobacterium avium complex [1]. Here, we report a cluster of confirmed, probable and possible HTL with occupational associations. To our knowledge, case 1 is the first confirmed case of occupational HTL in the literature.

A 30-yr-old hotel technician (case 1, table 1) developed episodes of cough, dyspnoea, fever and joint pain. His condition progressed with fatigue, weight loss and increasing dyspnoea. He was hospitalised at his third emergency visit. His oxygen tension was moderately reduced $(8.9 \mathrm{kPa})$. His C-reactive protein level was $16 \mathrm{mg} \cdot \mathrm{L}^{-1}$. A chest radiograph was normal but computed tomography showed ground-glass opacities without signs of embolism. He was dismissed after spontaneous remission 3 days later. He continued having some fatigue and dyspnoea on exertion. Fever, accentuated dyspnoea and cough relapsed twice after returning to work. Chest radiography showed diffuse interstitial infiltration and his diffusion capacity was reduced. Lymphocytosis was found in his bronchoalveolar lavage (BAL) fluid, and transbronchial biopsies showed interstitial inflammation and granuloma. HTL was diagnosed when growth of $M$. avium was found in BAL fluid and it became clear that he had cleaned the hotel's hot tub facility (facility 1) and its nylon filters with a pressure washer twice weekly.

Two colleagues who had substituted for the first hotel technician at servicing the bath facility during his sick leave also fell ill with similar symptoms (cases 2 and 3, table 1). They were eventually diagnosed with probable HTL but the workup was insufficient for a definite diagnosis.

Subsequently, four hotel technicians who had cleaned hot tubs and filters with a water hose were identified at two other hotels. Cases 4 and 5 at facility 2 (table 1) had experienced episodes of fever and dyspnoea after the cleaning procedure but had no subacute or chronic symptoms. M. avium was isolated from the BAL fluid of both patients. The other two hotel technicians (at facility 3, not shown in table 1) had been asymptomatic in association with their work but their sputum cultures were positive for M. avium.

The seven mentioned hotel technicians were included in this investigation. Five of them had symptoms associated with the hot tub exposure and are included in table 1 . In addition, only one other hotel technician had previously been involved in servicing the aforementioned bath facilities (facility 3). It is not known whether this person had experienced similar symptoms. One other person involved in the cleaning of filters at facility 1 on one single occasion developed a fever episode after exposure. Furthermore, one of the hotel technicians at facility 3 had used a hot tub at facility 3 with his girlfriend on two occasions and after these exposures, both had developed chills and fever. We have no indications of hot tub-related symptoms among guests at the hotels but such information is difficult to obtain.

The three spa facilities associated with our patients were similar in construction and maintenance. Their water disinfection systems combined a nylon-sheet particle filter with the use of ultraviolet light and hydrogen peroxide. After the nylon filters were replaced by sand filters, the three employees at facility 1 recovered spontaneously and sputum cultures of three of the employees at facilities 2 and 3 were negative (the fourth employee had no sputum sample taken). No treatment, such as antibiotics or corticosteroids, was given. After the refurbishment of the filters, all hotel technicians were able to resume servicing the baths without relapse or symptoms.

Three main risk factors for mycobacterial exposure at the spa facilities were identified: inadequate ventilation and wet storage of filters in facilities 1 and 2 and, most importantly, water aerosolisation during cleaning of filters in all facilities. The use of a pressure washer increased aerosolisation in facility 1 . Thus, exposure was assessed to be highest at facility 1 and lowest at facility 3 , corresponding to disease intensity.

Routine cultures from the baths for Pseudomonas and Legionella were negative. In conjunction with the patient investigations, endotoxin concentrations were not remarkably high in samples of air and water taken during cleaning of the filters. Water samples were analysed by nonquantitative culture for mycobacteria and $M$. avium was detected in all hot tub baths. Concentrations of M. avium between 140 and 16,000 CFU $\cdot \mathrm{mL}^{-1}$ were later determined in facility 2 when a method of quantitative culture was established [3].

The symptoms and findings consistent with HTL were judged to be caused by the hotel technicians' working environment. This conclusion was based on the thorough investigation of occupational hygienists and physicians, in which no other potentially pathogenic exposures outside the work place were found. The hotel technicians with symptoms recovered quickly and spontaneously when the source of the occupational exposure was removed. Furthermore, their improvement occurred without any changes in their personal daily lives (e.g. shower habits).

NTM, the pathogens behind HTL, are slowly growing, hydrophobic bacteria that can adhere to surfaces and produce a biofilm in, for example, water pipes [4]. They are resistant to most water disinfectants such as chlorine [4]. In a study from the USA [5], $M$. avium and other NTM were found in $72 \%$ of air and water samples randomly taken from 18 pool facilities. The concentrations were significantly lower in chlorine- and bromide-disinfected pools than in pools disinfected with hydrogen peroxide and ultraviolet light. 

on hot tubs

\begin{tabular}{|c|c|c|c|c|c|}
\hline & Case 1 & Case 2 & Case 3 & Case 4 & Case 5 \\
\hline \multicolumn{6}{|l|}{ Characteristics } \\
\hline Sex & Male & Male & Male & Male & Male \\
\hline Smoking history & Current & Former & Never & Former & Former \\
\hline Medical history & Healthy & Healthy & Healthy & Psoriasis & Hypertension \\
\hline Atopy & - & + & + & + & - \\
\hline Known immune deficiency & - & - & - & - & - \\
\hline Duration of maintenance of hot tub filters & 6 months & 4 months & 1 month & 8 yrs (sporadic) & $8 \mathrm{yrs}$ \\
\hline \multicolumn{6}{|l|}{ Symptoms } \\
\hline Duration months & 2 & 2 & 0.5 & 0.5 & 24 \\
\hline Recurrent influenza-like symptoms & + & + & + & + & + \\
\hline Fever & + & + & + & + & + \\
\hline Weight loss & + & - & - & - & - \\
\hline First diagnosis & Infection & Embolism & Infection & & \\
\hline \multicolumn{6}{|l|}{ Clinical investigations } \\
\hline $\mathrm{PO}_{2} \mathrm{kPa}$ & 8.9 & 7.9 & & 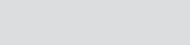 & \\
\hline Chest radiography: interstitial opacity & + & + & - & - & - \\
\hline HRCT: ground-glass opacity, nodular infiltrates & + & + & & & \\
\hline \multicolumn{6}{|l|}{$\mathrm{FVC}^{\#} \%$ pred } \\
\hline At diagnosis & 92 & 73 & 77 & 114 & \\
\hline After recovery & 121 & 102 & 108 & 108 & 95 \\
\hline \multicolumn{6}{|l|}{$\mathrm{FEV}_{1}{ }^{\#} \%$ pred } \\
\hline At diagnosis & 87 & 75 & 89 & 106 & \\
\hline M. avium in sputum culture & & & & + & \\
\hline
\end{tabular}

$\mathrm{PO}_{2}$ : oxygen tension; HRCT: high-resolution computed tomography; FVC: forced vital capacity; \% pred: \% predicted; FEV1: forced expiratory volume in $1 \mathrm{~s}$; $D L, C O$ : diffusing capacity of the lung for carbon monoxide; BAL: bronchoalveolar lavage; M. avium: Mycobacterium avium; OSAS: obstructive sleep apnoea syndrome;

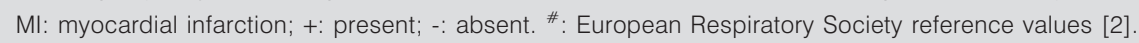

60-70 cases of HTL have been reported in immunocompetent users of hot tub baths, swimming pools and, occasionally, household showers. The clinical picture is similar to hypersensitivity pneumonitis [6]. Episodes of fever and dyspnoea are common, as well as a more gradual onset of dyspnoea and cough. As reviewed by SOOD et al. [7], all patients experience dyspnoea, while cough occurs in $80 \%$, fever in $55 \%$, chest discomfort in $25 \%$ and weight loss in $20 \%$ of patients.

There is an ongoing discussion of whether HTL should be regarded as hypersensitivity pneumonitis, infection, both or a disease entity of its own [8]. Chest radiography usually, but not always, shows scattered interstitial infiltrates or nodules.
High-resolution computed tomography is pathological in all patients, generally with ground-glass opacity and/or scattered, often centrilobular, nodular infiltrates [9]. Lung function tests show varying reduction of ventilation and diffusion capacity. Hypoxaemia is seen in half of the cases [7]. BAL shows lymphocytosis, and lung biopsy reveals granuloma, interstitial inflammation and elements of organising pneumonia with airspace filling fibroblastic plugs [10]. These findings correspond to those of hypersensitivity pneumonitis but in HTL, standardpanel precipitating antibody tests are negative and culture of respiratory samples reveal mycobacteria. Furthermore, the histology of HTL differ somewhat from that of hypersensitivity pneumonitis, with dominance of well-formed granulomas and 
less prominent interstitial inflammation [10]. According to the American Thoracic Society [1], a diagnosis of HTL can be made without histopathology if there is subacute onset of respiratory symptoms associated with hot tub exposure, positive mycobacterial culture from respiratory samples and water, and characteristic radiographic findings.

HTL may mimic other conditions and seems difficult to diagnose correctly, as illustrated by our cases that were initially diagnosed as infection, pulmonary embolism and hypersensitivity pneumonitis. Patients often fail to associate their symptoms with the hot tub exposure. Thus, hot tub exposure should be inquired in patients presenting with respiratory and constitutional symptoms of uncertain aetiology. If our first case had not been persistent in his notion that his symptoms were work related and had he not been thoroughly investigated, neither his nor his colleagues' diagnoses would have been revealed.

Complete avoidance of exposure is paramount for the recovery of patients with HTL and many patients recover within weeks without other treatment $[1,6,7]$. Treatment with steroids is considered to hasten recovery in advanced cases. Antimycobacterial therapy may be given but the prognosis is good even without such treatment. No chronic or mortal cases have been reported [1, 7].

When our first patients were diagnosed in 2007, there were no reports of occupational HTL published. A case of suspected HTL in a 17-yr-old female swimming instructor was reported in New Zealand in 2008 [11]. An outbreak of diffuse granulomatous lung disease among life guards was previously reported from an indoor swimming pool with many water-spraying devices [12]. High concentrations of endotoxins were found but the aetiology of the outbreak was not established. In subsequent articles, HTL has been suggested to explain the outbreak [4, 7].

Considering the abundance of NTM in our environment, the medical risks of exposure may generally be regarded as small. However, we recommend that aerosol-producing procedures are avoided in the cleaning of equipment likely to carry mycobacteria. Pressure washers should not be used.

\section{Harald Fjällbrant*,\#, Magnus Akerstrom ${ }^{\star}$, Erik Svensson ${ }^{+, \S}$ and Eva Andersson $₫$}

*Institute of Medicine, Dept of Respiratory Medicine and Allergology, Sahlgrenska Academy, University of Gothenburg, "Dept of Occupational and Environmental Medicine, Sahlgrenska Academy, University of Gothenburg, ' Institute of Biomedicine, Dept of Bacteriology, Sahlgrenska Academy, University of Gothenburg, Gothenburg, and \#AstraZeneca

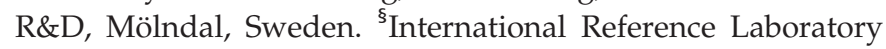

of Mycobacteriology, Statens Serum Institut, Copenhagen, Denmark.

Correspondence: H. Fjällbrant, AstraZeneca R\&D Mölndal, SE-431 83 Mölndal, Sweden. E-mail: harald.fjallbrant@astra zeneca.com.

Statement of Interest: H. Fjällbrant is an employee of AstraZeneca.

Provenance: Submitted article, peer reviewed.

\section{REFERENCES}

1 Griffith DE, Aksamit T, Brown-Elliott BA, et al. An official ATS/ IDSA statement: diagnosis, treatment, and prevention of nontuberculous mycobacterial diseases. Am J Respir Crit Care Med 2007; 175: 367-416.

2 Quanjer PH, Tammeling GJ, Cotes JE, et al. Lung volumes and forced ventilatory flows. Report working party standardization of lung function tests, European Community for Steel and Coal. Official statement of the European Respiratory Society. Eur Respir J 1993; 6: Suppl. 16, 5-40.

3 Svensson E, Akerstrom M, Andersson E. Quantitative analyses of mycobacteria in water: adapting methods in clinical laboratories. J Microbiol Methods 2011; 87: 114-115.

4 Falkinham JO 3rd. Nontuberculous mycobacteria in the environment. Clin Chest Med 2002; 23: 529-551.

5 Glazer CS, Martyny JW, Lee B, et al. Nontuberculous mycobacteria in aerosol droplets and bulk water samples from therapy pools and hot tubs. J Occup Environ Hyg 2007; 4: 831-840.

6 Hanak V, Kalra S, Aksamit TR, et al. Hot tub lung: presenting features and clinical course of 21 patients. Respir Med 2006; 100: 610-615.

7 Sood A, Sreedhar R, Kulkarni P, et al. Hypersensitivity pneumonitis-like granulomatous lung disease with nontuberculous mycobacteria from exposure to hot water aerosols. Environ Health Perspect 2007; 115: 262-266.

8 Daito H, Kikuchi T, Sakakibara T, et al. Mycobacterial hypersensitivity pneumonitis requires TLR9-MyD88 in lung CD11b+CD11c+ cells. Eur Respir J 2011; 38: 688-701.

9 Hartman TE, Jensen E, Tazelaar HD, et al. CT findings of granulomatous pneumonitis secondary to Mycobacterium aviumintracellulare inhalation: "hot tub lung". AJR Am J Roentgenol 2007; 188: 1050-1053.

10 Agarwal R, Nath A. Hot-tub lung: hypersensitivity to Mycobacterium avium but not hypersensitivity pneumonitis. Respir Med 2006; 100: 1478.

11 Yu TC, Ahmed R, Yap E, et al. Dyspnoea in a 17-year-old swim instructor: a diagnosis of hot tub lung. NZ Med J 2008; 121: 78-80.

12 Rose CS, Martyny JW, Newman LS, et al. "Lifeguard lung": endemic granulomatous pneumonitis in an indoor swimming pool. Am J Public Health 1998; 88: 1795-1800.

DOI: $10.1183 / 09059180.00002312$ 